\title{
Polysaccharides from Dolichos biflorus Linn and Trachyspermum ammi Linn seeds: isolation, characterization and remarkable antimicrobial activity
}

\author{
Shibani Basu', Manojit Ghosh², Rupam Kumar Bhunia ${ }^{3}$, Jhuma Ganguly ${ }^{1 *}$ and Bimal K. Banik ${ }^{4^{*}}$
}

\begin{abstract}
Polysaccharides are structurally complex and essential constituents of life, and therefore, studies directed to these kinds of molecules have received scientific attention. Despite an easy availability of Dolichos biflorus Linn and Trachyspermum ammi (Linn) seeds isolation, characterization and antimicrobial studies of polysaccharides derived from these two natural sources have not been investigated. Therefore, we report here isolation of polysaccharides, their purification and characterization from Dolichos biflorus Linn and Trachyspermum ammi (Linn) seeds. Gel permeation chromatography, GC-MS, SEM, XRD, EDX and FT-IR analyses show the presence of three pentose sugar such as D-ribose, D-arabinose, D-xylose and hexose sugar such as D-mannose, D-galactose and D-glucose. Unprecedented antimicrobial activity of these polysaccharides against Gram positive bacteria such as Staphylococcus aureus and Bacillus subtilis and Gram negative bacteria such as Escherichia coli and Pseudomonas aeruginosa are established.
\end{abstract}

Keywords: Polysaccharides, Structural elucidation, Chemical characterization, Physiochemical identification, Antimicrobial activity

\section{Introduction}

There has been tremendous interest in the use of medicinal plants in developed as well as developing countries, because compounds obtained from medicinal plants have been shown to be effective sources of therapeutic agents, without undesirable side effects $[1,2]$. Polysaccharides are very crucial since they have tremendous medicinal values. Current studies have proved that the structures of polysaccharides are closely related to their biological activities [3]. So elucidation of their structures is a fundamental objective for understanding structure-activity relationships and cause of these biological activities.

We report here an analysis of the structural composition of purified polysaccharides extracts and chemical

\footnotetext{
*Correspondence: jhumaiiest@gmail.com; bimalbanik10@gmail.com 1 Department of Chemistry, Indian Institute of Engineering Science and Technology, Shibpur, Howrah, West Bengal, India

${ }^{4}$ Research \& Education Development, Community Health Systems of South Texas, 3135 South Sugar Road, Edinburg, TX 78539, USA Full list of author information is available at the end of the article
}

nature of Trachyspermum ammi (Linn.) Sprague and Dolichos biflorus Linn in detail. Literature is found less secured on the elucidation of their structures of purified extracts of these species and studies of their significant biological activity.

Trachyspermum ammi (Linn) Sprague (Ajowan), belongs to the family of Apiaceae. Also known as bishop's weed, it is an aromatic spice closely resembling thyme in flavor [4]. It is a native of Egypt and is distributed in the Mediterranean region and South-west Asia. It has long being used as the principal source of thymol $T$. ammi seeds and is employed as an antiseptic, aromatic, carminative and antioxidant source [5]. Its oil is used in the preparation of lotions and ointments in cosmetics industries and as a spice in many food preparations [6]. It has been reported to possess strong insecticidal activity, bronchodilatory effect on asthmatic airways and analgesic effect [7-9].

Dolichos biflorus Linn (Horsegram or Kulthi) is well known throughout India as a draught resistant crop. It 
also enriches the soil with nitrogen. So after harvesting the seeds, the soil can be ploughed with green manure [10]. Dolichos biflorus is a well known medicinal plant for its folk-medicinal properties. In herbal medicine, the seeds of it are mainly used as tonic, astringent, diuretic, and are also recommended in asthma, bronchitis, urinary discharges, hiccoughs, heart trouble and other diseases of the brain [11-13].

After isolation, both samples from the seeds of T. ammi and $D$. biflorus were purified, analyzed and compared by chemical and physico-chemical route. The percentage of active ingredients in the purified extract from the natural samples is dependent on geographical distribution as well as the environmental conditions such as temperature, rainfall, altitude, and hr of sun light exposure.

\section{Materials and methods}

\section{Sample preparation}

Seeds of Dolichos biflorus Linn and Trachyspermum ammi (Linn) Sprague were obtained from West Bengal, India and identified (Fig. 1, Table 1). Seed samples were prepared by extracting $10 \mathrm{gm}$ of seeds of each sample separately in $50 \mathrm{~mL}$ of water and stirring for $48 \mathrm{~h}$ at room temperature $\left(25^{\circ} \mathrm{C}\right)$. The samples and named as TAE $(T$. ammi water extract) and DAE (D. biflorus water extract).

\section{Isolation and purification of water soluble carbohydrate}

The crude aqueous extracts were purified by gel permeation chromatography (GPC). The freeze-dried samples were dissolved in deionized water and applied to a Sephadex G-25 column $(600 \times 16 \mathrm{~mm})$ that had previously been equilibrated with Mili-Q water at a flow rate of $0.3 \mathrm{~mL} \mathrm{~min}-1$ and the column was then eluted with of Mili-Q water. Collected fractions were identified by phenol-sulphuric acid assay at $490 \mathrm{~nm}$ for the estimation of neutral sugar [14].

\section{Compositional analysis}

The polysaccharides were hydrolyzed by TFA and derived by "acetic anhydride-pyridine" method. The derivatives were used to determine the monosaccharide compositions, because monosaccharides, in these conditions, were converted to volatile substances and therefore, they were easy to be detected by gas chromatographymass spectrometry. Analytical GC and GC-MS were performed for quantitative and qualitative analysis of monosaccharides present in the purified fraction using alditol acetate derivatives with inositol as an internal standard [15]. Samples $(200 \mu \mathrm{g})$ were hydrolyzed with 2N TFA (trifluoroacetic acid) at $120{ }^{\circ} \mathrm{C}$ for $2 \mathrm{~h}, \mathrm{NaBH}_{4}$ (sodium borohydride) was used for reduction followed by $1 \mathrm{~h}$ incubation at $100{ }^{\circ} \mathrm{C}$ with acetic anhydride and pyridine (1:1). The hydrolyzed monosaccharides were then extracted with DCM (dichloromethane), analyzed on Agilent 6820 gas chromatograph equipped with HP-5 fused silica capillary column $(30 \mathrm{~m} \times 0.25 \mathrm{~mm}$ I.D). Mass spectra quantified on GC 7890A series equipped with MS 7000 GC/MS triple quad using DB-5 silica capillary
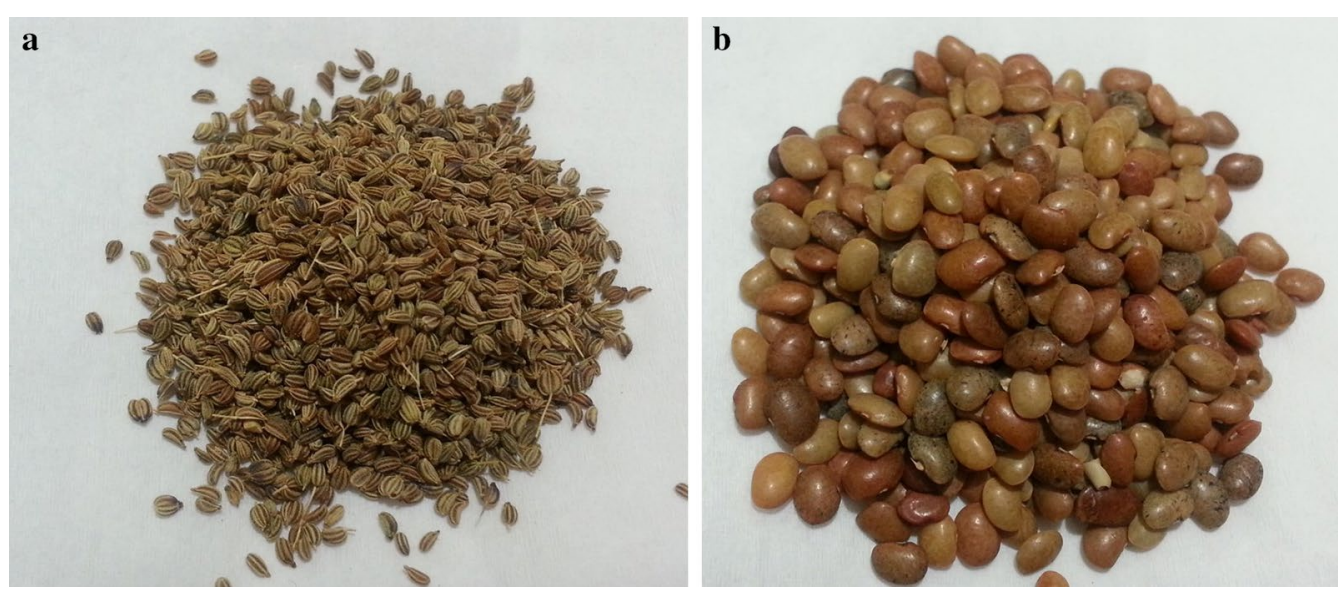

Fig. 1 Seed of Trachyspermum ammi (Linn) Sprague (a) and seed and Dolichos biflorus Linn (b)

Table 1 Identification and authentication of samples

\begin{tabular}{lllll}
\hline Sl. no. & Specimen/sample no. & Scientific name & Common name & Family name \\
\hline 1 & J.G.1 & Trachyspermum ammi (Linn) Sprague & Ajwain, ajoan, carom & Apiaceae; Alt. Umbelliferae \\
2 & J.G.2 & Dolichos biflorus Linn & Horse-gram, kulthi, hurali, Madras gram & Fabaceae; Alt. Leguminosae \\
\hline
\end{tabular}


column (30 $\mathrm{m} \times 0.25 \mathrm{~mm}$ I.D). Nitrogen was used as the carrier gas $\left(1.5 \mathrm{~mL} \mathrm{~min}{ }^{-1}\right)$. Detection was done by FID $\left(300{ }^{\circ} \mathrm{C}\right)$ and identification by mass spectrometry held at $250^{\circ} \mathrm{C}$.

\section{FT-IR analysis}

The major structural groups of purified extracts were detected using Fourier transform infrared spectroscopy (FT-IR). The FT-IR spectra were recorded in the region of $4000-400 \mathrm{~cm}^{-1}$ on a JASCO, FT/IR-460 PLUS using KBr pellet method. Background correction was made using a reference blank $\mathrm{KBr}$ pellet. The purified polysaccharides were ground with $\mathrm{KBr}$ powder by mortar and pestle. The mixture was then pressed into pellets for FTIR measurement in the frequency range of $4000-500 \mathrm{~cm}^{-1}$.

\section{SEM and EDX analysis}

The samples were used for Scanning electron microscopy (SEM) analysis by fabricating a drop of suspension onto a clean electric stubs and allowing the solvent (i.e. water, methanol) to completely evaporate with platinum coating. The morphology of the purified extracts was observed on a ZEISS EVO 18 electron microscope with an accelerated voltage of $10-20 \mathrm{kV}$.

Elemental analysis of purified extracts was carried out using energy dispersive X-ray spectroscopy (EDX). The EDX of the purified extracts were measured by ZEISS EVO 18 electron microscope.

\section{XRD analysis}

X-ray diffraction (XRD) analysis of drop-coated films of extracts was prepared for the study of phase and nature. A Bruker D-8 Advanced X-ray diffractometer with scanning range from $2 \theta=20^{\circ}$ to $80^{\circ}$ and $\mathrm{Ni}$-filtered $\mathrm{Cu} \mathrm{K \alpha}$ radiation with wavelength $1.540598 \AA$ used for this characterization.

\section{TGA-DTA analysis}

Thermal analysis gives properties like enthalpy, thermal capacity, mass changes and the coefficient of heat expansion. TGA and differential scanning calorimetric (DTA) analysis were carried out by Perkin Elmer SII, Diamond TG/DTA machine. The samples were heated to $600{ }^{\circ} \mathrm{C}$ at a heating rate of $10{ }^{\circ} \mathrm{C} \mathrm{min}{ }^{-1}$, under flowing nitrogen of $10 \mathrm{~mL} \mathrm{~min}^{-1}$.

\section{Study of antimicrobial activity}

The disc diffusion method was applied to evaluate the antimicrobial activity with four human pathogenic bacteria. Gram positive bacteria such as Staphylococcus aureus, Bacillus subtilis, Gram negative bacteria such as Escherichia coli, Pseudomonas aeruginosa were used in this study. $100 \mathrm{~mL}$ conical flask of nutrient broth was inoculated with the test organisms and incubated at $37^{\circ} \mathrm{C}$ for overnight. By using a sterile pipette, $0.6 \mathrm{~mL}$ of the broth culture of each test organism was added to $60 \mathrm{~mL}$ of molten agar, which was cooled at $45^{\circ} \mathrm{C}$, mixed well and poured into a sterile Petri plate. Normal saline water was used as negative control and Tetracycline was used as positive control in comparison with test organisms. Then, the plates were left at room temperature for $2 \mathrm{~h}$ to allow diffusion of the test sample and incubated face upwards at $37^{\circ} \mathrm{C}$ for overnight.

\section{Results and discussion}

Isolation and purification by gel permeation chromatography (GPC) on a Sephadex G-25 column $(600 \times 16 \mathrm{~mm})$ obtained UV actives region (at $490 \mathrm{~nm}$ ) peaks for the phenol sulfuric acid assay (Fig. 2) from TAE and DAE (extracts were eluted with deionized water). As shown in Fig. 2, absorbance at $490 \mathrm{~nm}$ was observed, confirming that the isolated fractions were mainly polysaccharide rich.
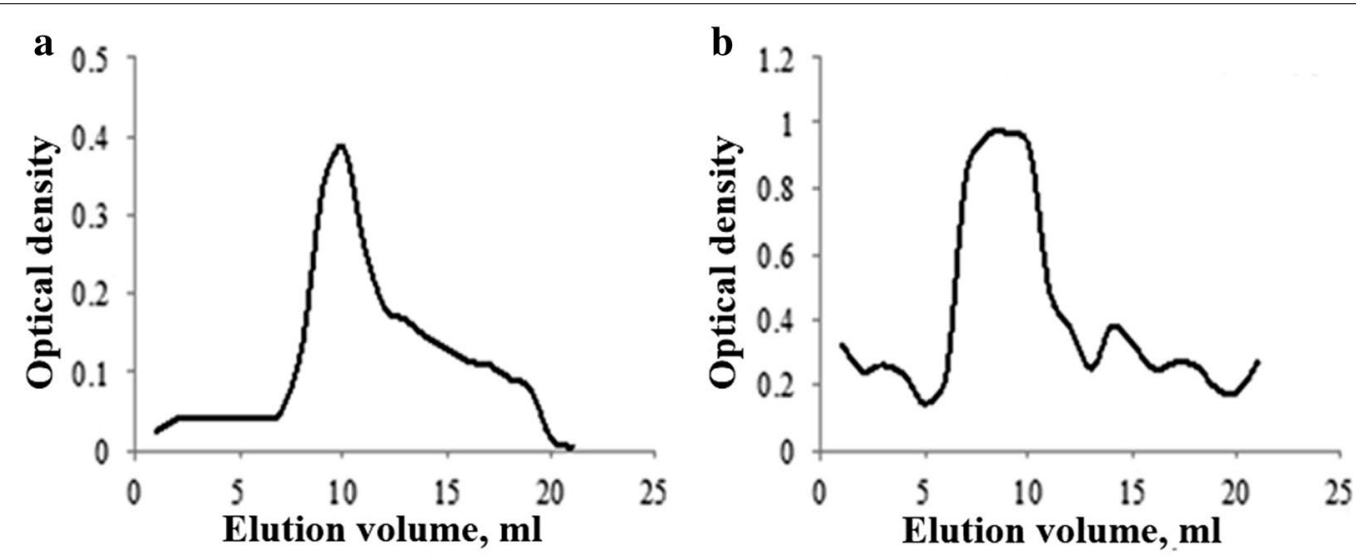

Fig. 2 UV spectrophotometry analysis (the UV actives region at $490 \mathrm{~nm}$ ) for the purity of water soluble polysaccharides of T. ammi (Linn) Sprague (a) and D. biflorus Linn. (b) purified by gel permeation chromatography 
GC-MS analysis determined six kinds of monosaccharides in TAE and four kinds of monosaccharide in DAE by comparing with standard sugar peak (Fig. 3). The GC and GC-MS analyses showed the presence of three pentose sugars such as D-ribose, D-arabinose, D-xylose and three hexose sugars such as D-mannose, D-galactose and D-glucose in TAE (Fig. 4). Importantly, the molecular ratios of the monosaccharide constituents in TAE were found to be present at 6:7:3:38.2:35.1:10.5 (Table 2).

On the other hand, by the GC-MS analysis in DAE (Fig. 5) confirmed the presence of D-arabinose, D-xylose as pentose sugar and D-mannose, D-galactose, D-glucose as hexose sugar with the molecular ratio of 2:1: 2:37:58 respectively (Table 3 ).

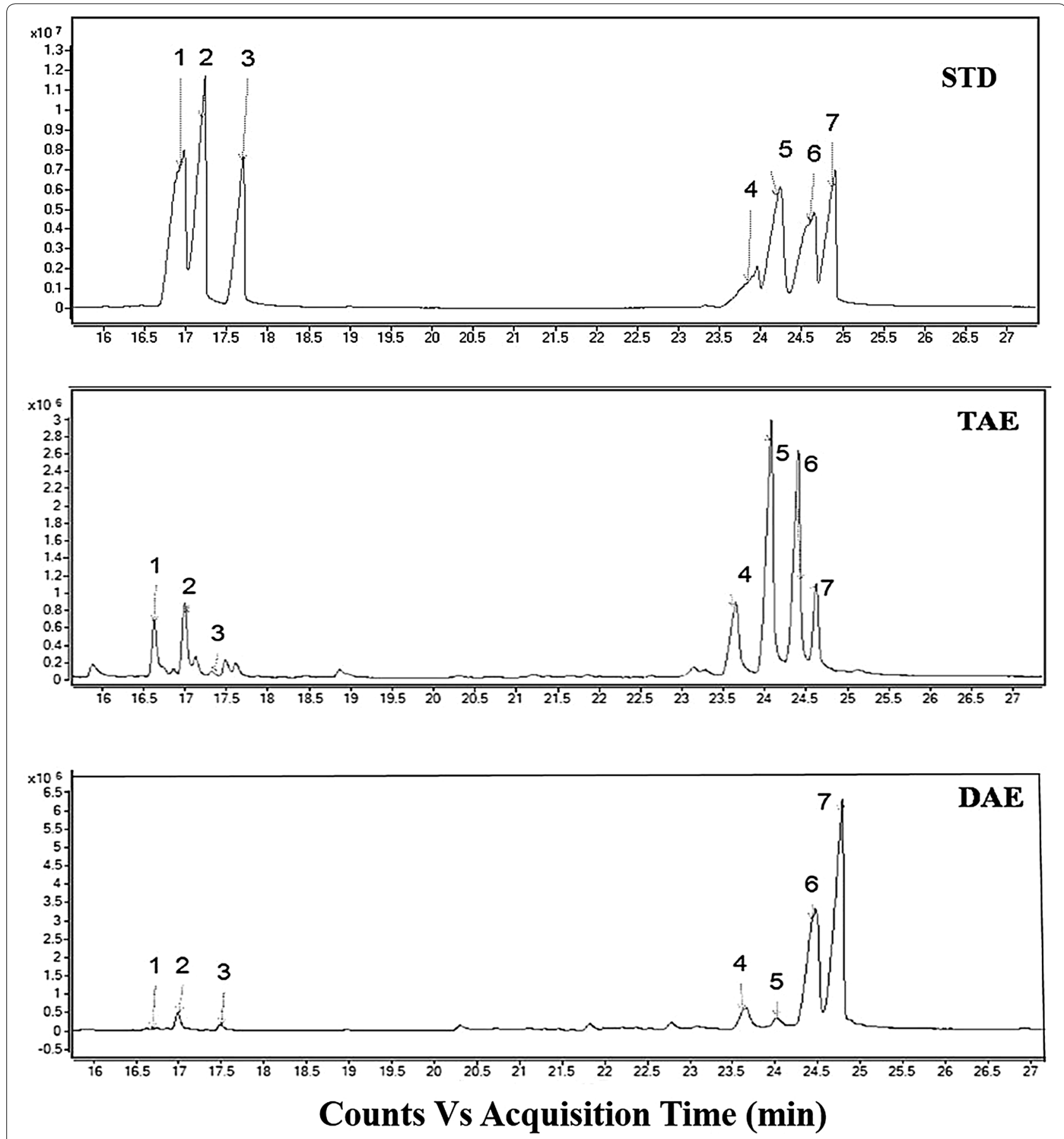

Fig. 3 GC spectrum of standard sugar mixture (STD), TAE and DAE containing ribose (1), arabinose (2), xylose (3), inositol (4), mannose (5), glucose (6), galactose (7) 


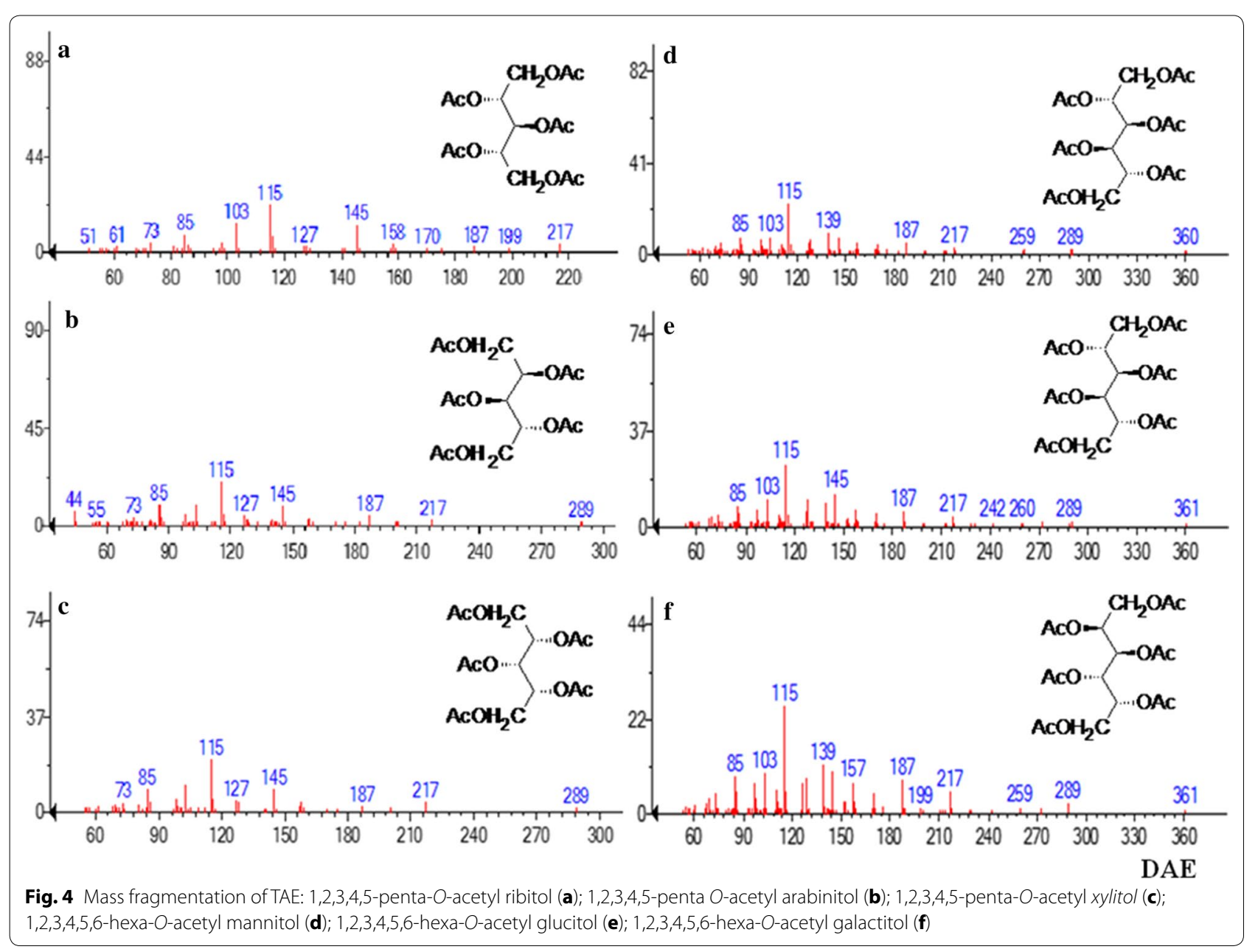

Table 2 Molecular ratio of monosaccharide mixture from water soluble polysaccharides

\begin{tabular}{llllllll}
\hline Sample name & \% of CHO & & \multicolumn{2}{l}{ Compound presents \# (mol\%) } \\
\cline { 3 - 8 } & & Arabinose & Ribose & Xylose & Mannose & Galactose & Glucose \\
\hline TAE & 70.05 & 6 & 7 & 3 & 38.2 & 35.1 & 10.5 \\
DAE & 70.09 & 2 & - & 1 & 2 & 37 & 58 \\
\hline
\end{tabular}

The functional group of purified sample was determined by FTIR spectroscopy using $\mathrm{KBr}$ pellet method (Fig. 6). The purified polysaccharides were ground with $\mathrm{KBr}$ powder and then pressed into pellets for FTIR measurement in the frequency range of $4000-500 \mathrm{~cm}^{-1}$. Dried TAE and DAE (water extract/methanol extract) showed a peak at $3317 \mathrm{~cm}^{-1}$ due to the stretching of the $\mathrm{N}-\mathrm{H}$ bond of amino groups and indicative of bonded hydroxyl $(-\mathrm{OH})$ groups alcohols and phenolic compounds. The absorption bands at $2926 \mathrm{~cm}^{-1}$ that appeared in spectrums were due to the stretching frequency of $-\mathrm{CH}_{3}$ groups. A peak at $1733 \mathrm{~cm}^{-1}$ in dried DAE indicated the presence of characteristic $\mathrm{C}=\mathrm{O}$ stretching frequency of the carboxylic acid group. However, no such peak was present in TAE indicating the absence of $-\mathrm{COOH}$ group in it. It also shows absorption bands at 1633 and $1564 \mathrm{~cm}^{-1}$ for carbonyl stretching vibration (amide-I) or $\mathrm{C}=\mathrm{C}$ groups/aromatic rings, $\mathrm{N}-\mathrm{H}$ stretching vibration (amide-II), respectively for amide linkages of the proteins present in it, while medium broad band at $1400 \mathrm{~cm}^{-1}$ is the $\mathrm{C}-\mathrm{N}$ stretching mode of aromatic amine group [16]. The absorption band at $989 \mathrm{~cm}^{-1}$ was due to the C-O-C vibrations of proteins/polysaccharides present in the extract [17]. The band at $840 \mathrm{~cm}^{-1}$ was assigned due to 


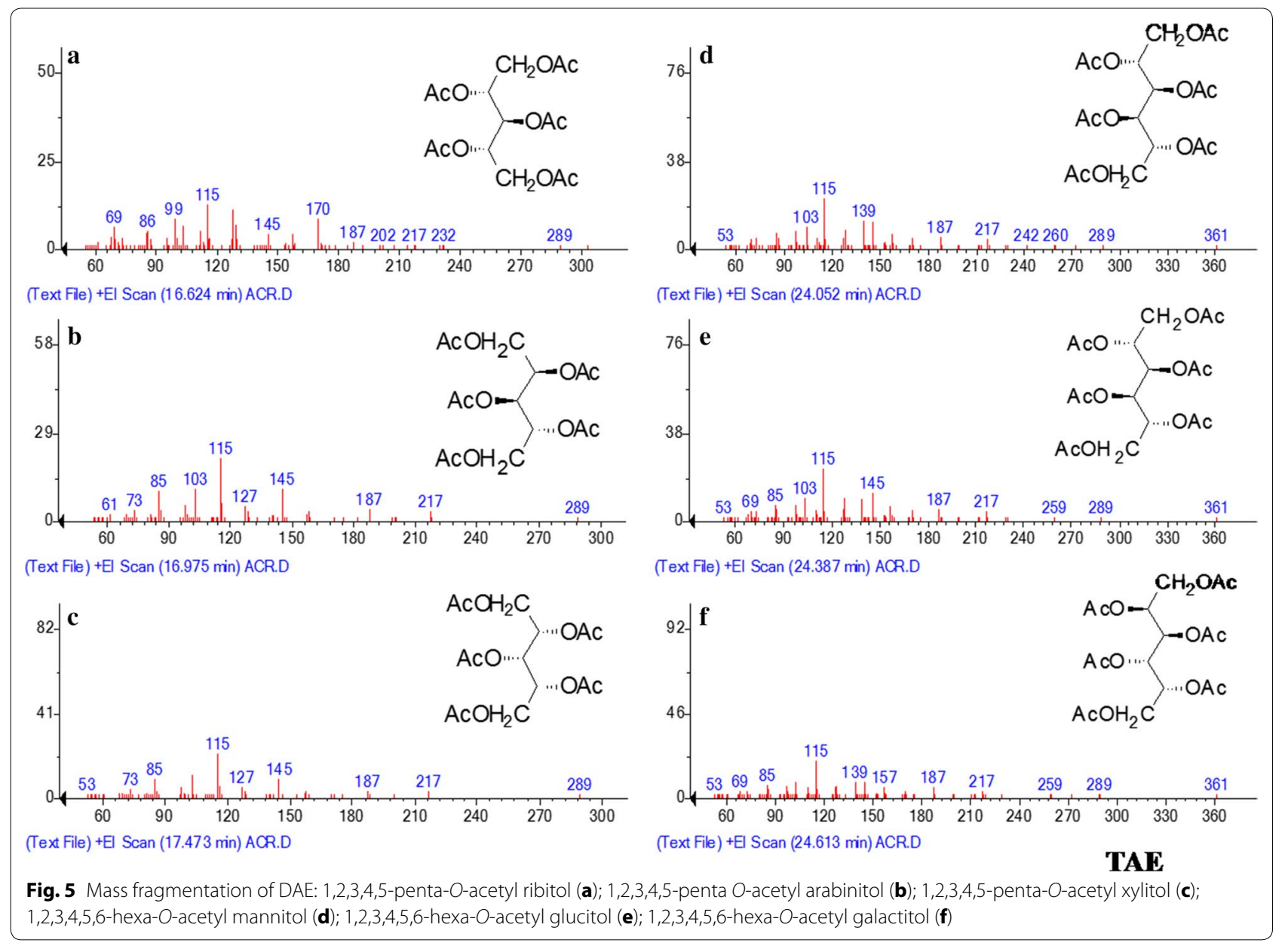

Table 3 Elemental study of purified extracts by EDX

\begin{tabular}{lllllllllll}
\hline Element & Sample & $\mathbf{C}$ & $\mathbf{O}$ & $\mathbf{M g}$ & $\mathbf{P}$ & $\mathbf{S}$ & $\mathbf{C l}$ & $\mathbf{K}$ & $\mathbf{C a}$ & $\mathbf{S n}$ \\
\hline Weight\% & TAE & 45.67 & 46.76 & 0.54 & 0.30 & 0.27 & 0.91 & 3.67 & 1.35 & 0.53 \\
& DAE & 55.54 & 41.40 & - & 0.30 & - & 0.18 & 2.57 & - & - \\
\multirow{2}{*}{ Atomic\% } & TAE & 54.93 & 42.22 & 0.32 & 0.14 & 0.12 & 0.37 & 1.36 & 0.49 & 0.06 \\
& DAE & 63.41 & 35.49 & - & 0.13 & - & 0.07 & 0.90 & - & - \\
\hline
\end{tabular}

the in plane and out of plane bending for benzene ring. This indicated that extracts are surrounded by some proteins and metabolites such as alkaloids and terpenoids that have functional groups of amines, alcohols, ketones, aldehydes, and carboxylic acids.

The morphology of the samples was observed under scanning electron microscope (SEM) with an accelerated voltage of $15 \mathrm{kV}$. SEM analyses showed that the purified samples were compact in nature with an uneven surface (Fig. 7). Also, it was not porous as observed in the samples extracted from TAE and DAE. The elemental analysis estimated that (Fig. 8) carbon, oxygen, magnesium, phosphorus, sulfur, chlorine, potassium, calcium, and tin are the elements mainly found in both extract (Table 3 ).

XRD analysis of samples represented the amorphous structure of the samples. As shown in Fig. 9, samples showed large hump distributed in a wide range $(2 \theta)$ instead of high intensity narrower peaks with interplanner spacing (d-spacing) of 3.99 and 3.88 revealing the amorphous structure of the samples.

Thermal gravimetric analysis or (TGA) measured changes in weight in relation to changes in temperature. 


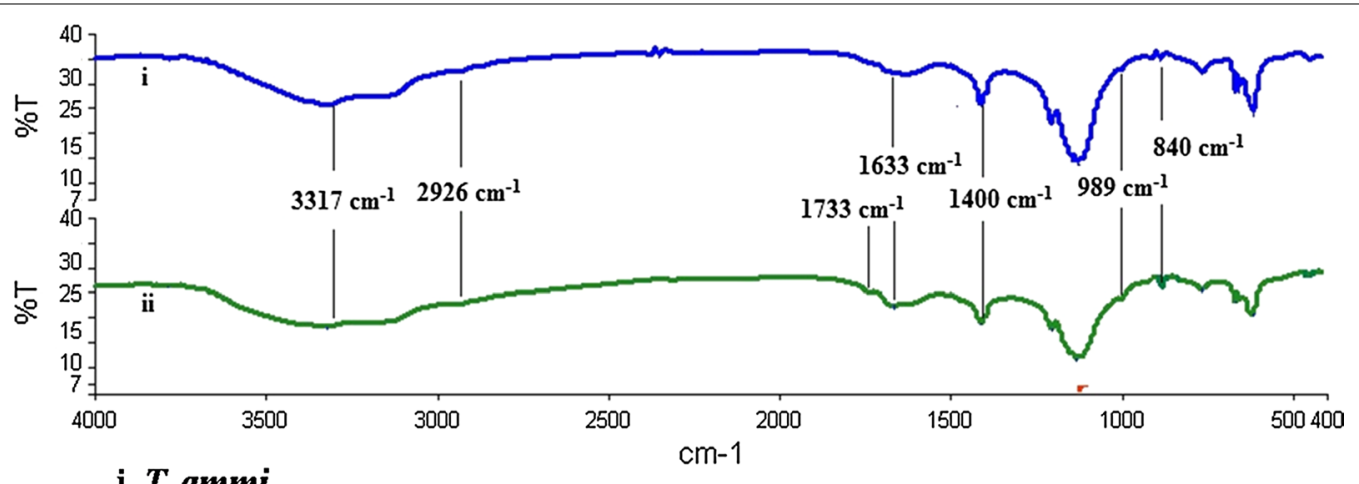

\section{i. T. ammi \\ ii. D. biflorus}

Fig. 6 FTIR spectroscopy of purified sample
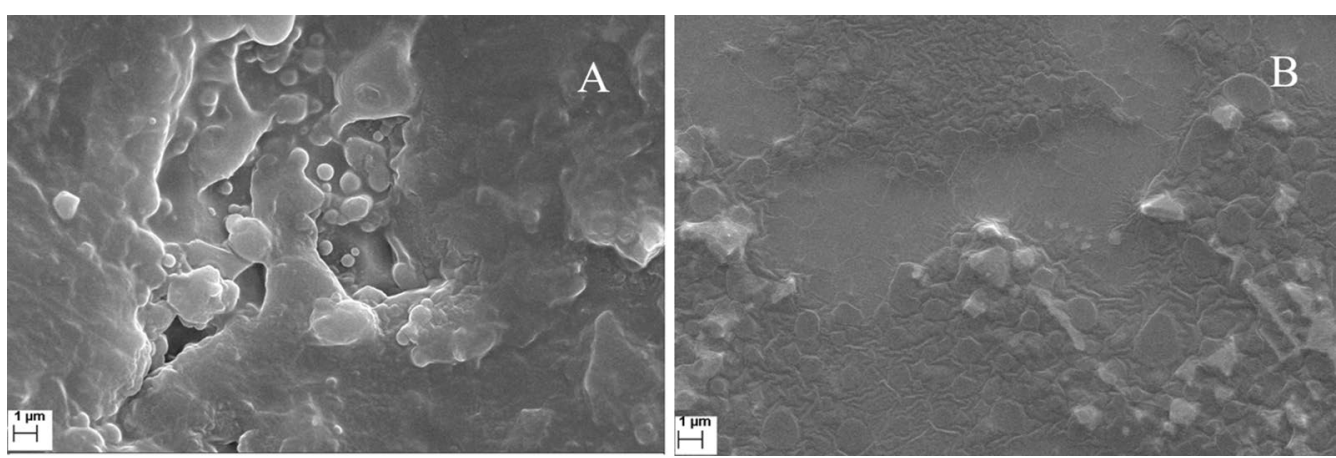

Fig. 7 SEM images of TAE (A) and DAE (B) purified fraction
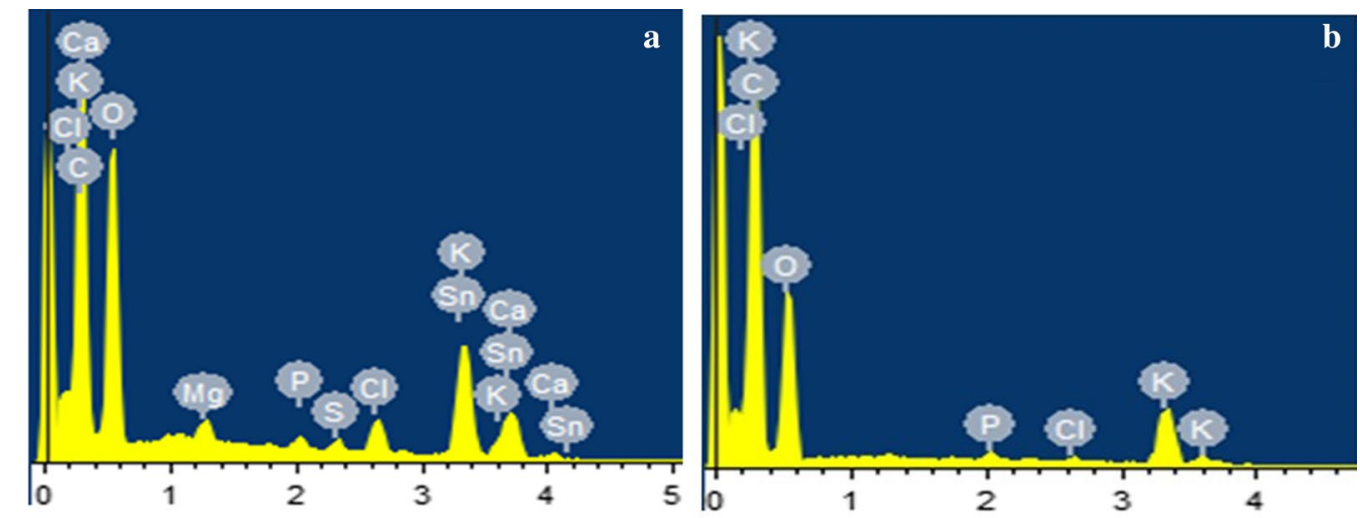

Fig. 8 EDX of purified TAE (a) and DAE (b)

TGA is commonly used to determine selected characteristics of materials that exhibit either mass loss or gain due to decomposition, oxidation, or loss of volatiles e.g. moisture. The TGA analysis showed degradation of TAE and DAE in two well-defined steps for both materials
(Fig. 10). A total of 10.04 and $10.07 \%$ of weight loss for the first and 75.63 and $74.10 \%$ for the second step were recorded for samples TAE and DAE respectively. As discussed, weight loss during the first step below $100{ }^{\circ} \mathrm{C}$ indicated removal of moisture. From the results of DTA it 


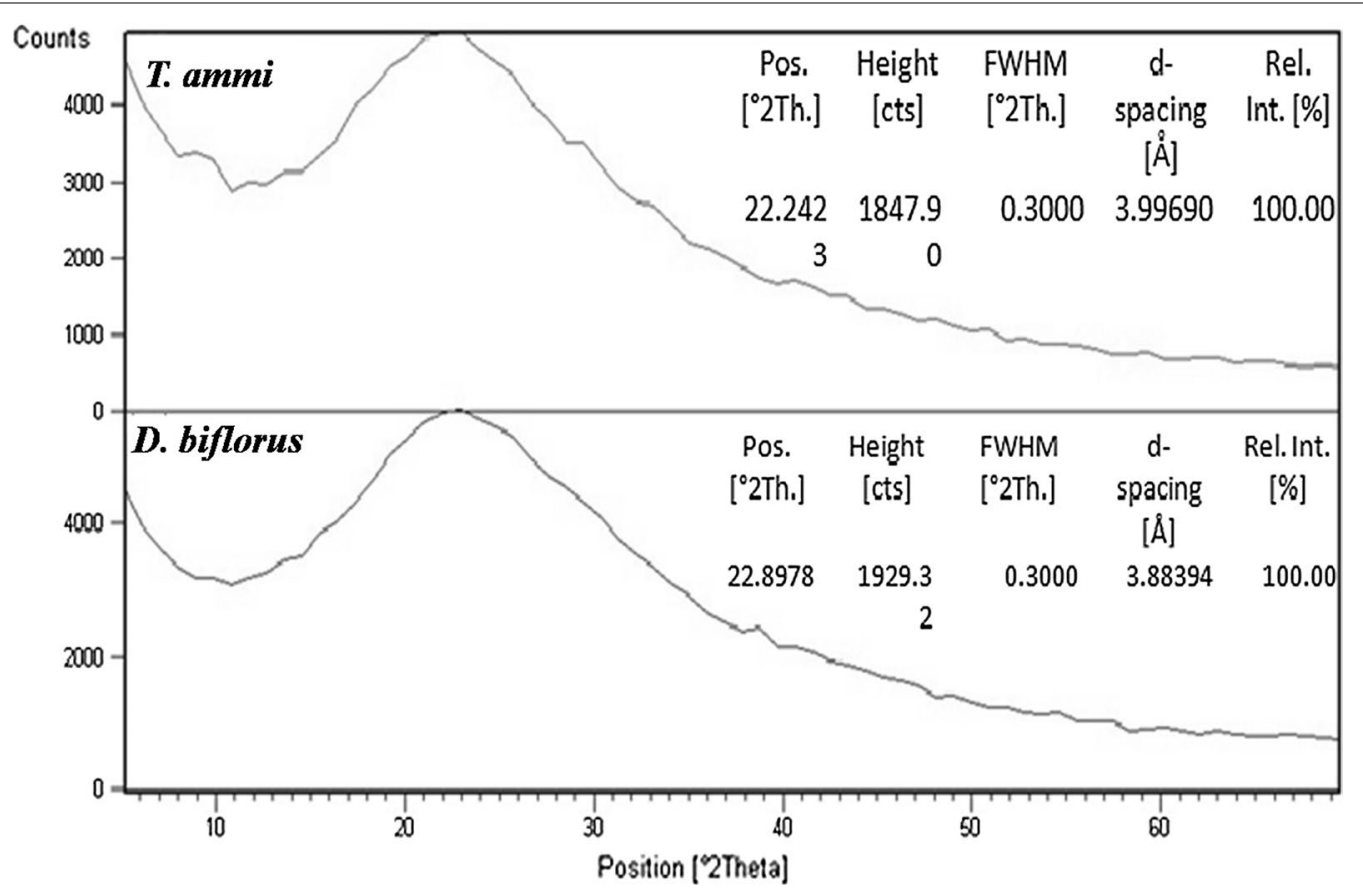

Fig. 9 XRD profile of T. ammi (Linn) Sprague and D. biflorus Linn purified fraction of water extract
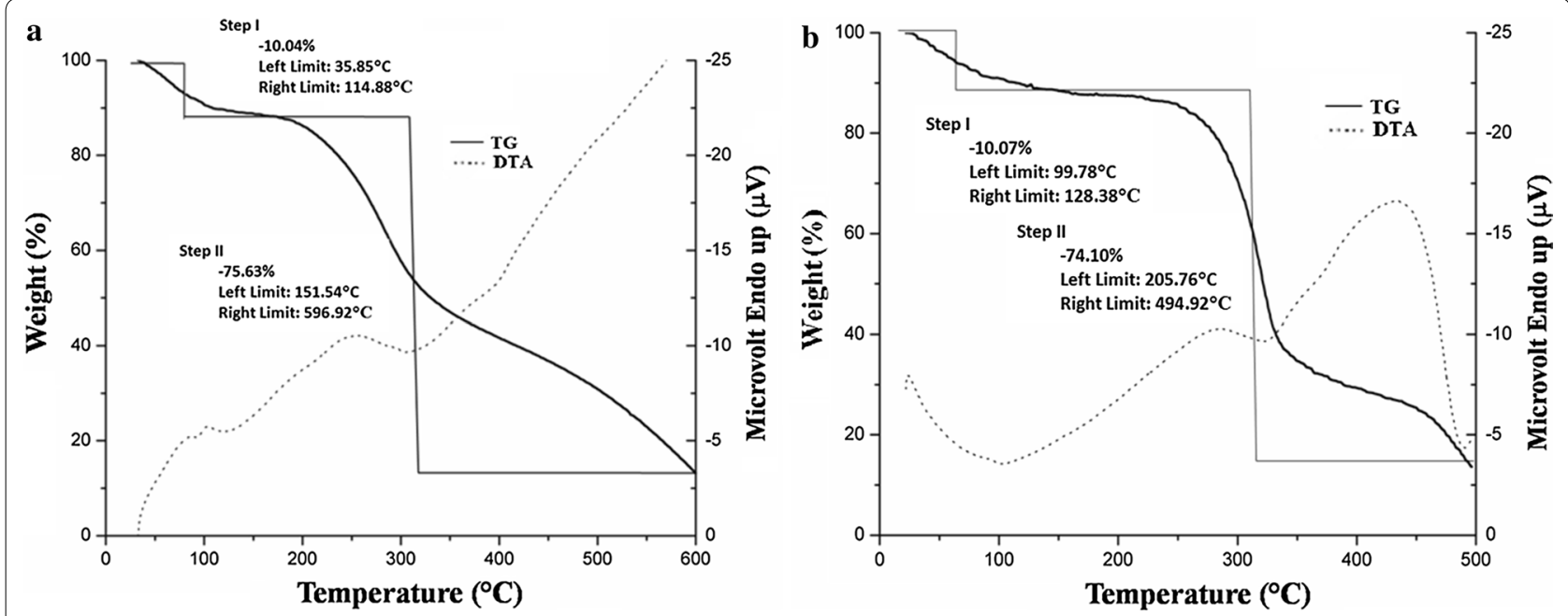

Fig. 10 TGA and DTA response of (a) T. ammi and (b) D. biflorus purified extracts

can be clearly noticed that all the reactions were exothermic in nature.

Importantly it was detected that TAE and DAE have antibacterial activity (Fig. 11) against gram positive bacteria such as Staphylococcus aureus, Bacillus subtilis. In well diffusion method, TAE showed a maximum zone of inhibition against Staphylococcus aureus $(1.9 \mathrm{~cm})$ whereas DAE showed $1 \mathrm{~cm}$ zone of inhibition. A positive control (tetracycline) was used for comparative data and it showed $3.7 \mathrm{~cm}$ zone of inhibition. On the other hand, TAE showed zone of inhibition as 1.8 and $1.7 \mathrm{~cm}$ against Bacillus subtilis whereas, DAE showed $1.4 \mathrm{~cm}$ zone of inhibition. Extracts of both TAE and DAE showed significant antimicrobial activity with comparison to positive control (tetracycline) which showed $2.9 \mathrm{~cm}$ zone of inhibition. The zones of inhibition (cm) (Fig. 12) were reported in Table 4. 


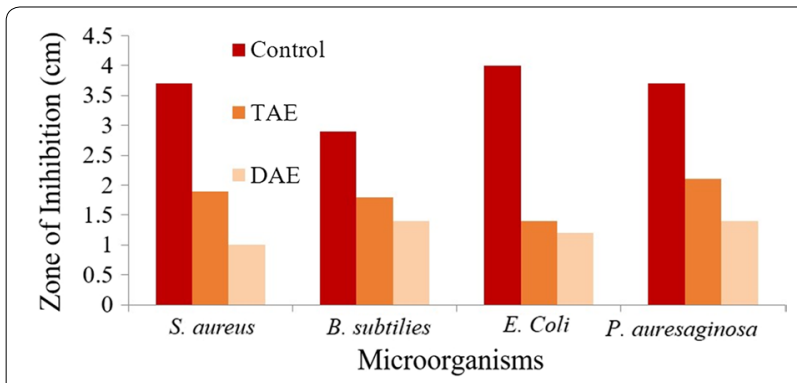

Fig. 11 Antimicrobial activity against human pathogenic bacteria of TAE and DAE in comparison with positive control as tetracycline

The above results were very encouraging and deserved further attention. It was gratifying to note that TAE and DAE also have antibacterial activity against Gram negative bacteria such as Escherichia coli and Pseudomonas aeruginosa. TAE showed a zone of inhibition as $1.4 \mathrm{~cm}$ and DAE showed $1.2 \mathrm{~cm}$ against Escherichia coli. Thus, the extracts of both T. ammi and D. biflorus showed antimicrobial activity in comparison to positive control (tetracycline) which showed $4 \mathrm{~cm}$ zone of inhibition. TAE showed the highest zone of inhibition against Pseudomonas aeruginosa $(2.1 \mathrm{~cm})$ among rest of microorganisms tested. DAE showed $1.4 \mathrm{~cm}$ zone of inhibition in comparison to positive control (tetracycline) which showed $3.5 \mathrm{~cm}$ zone inhibition. The molecular ratios of monosaccharide mixtures present in water soluble polysaccharide had significant effects on microbial activities.
Table 4 Antimicrobial activity of purified extracts

\begin{tabular}{lllr}
\hline Microorganism & \multicolumn{3}{l}{ Zone of inhibition $(\mathbf{c m})$} \\
\cline { 2 - 4 } & Control & \multicolumn{1}{l}{ TAE } & \multicolumn{1}{c}{ DAE } \\
\hline Staphylococcus aureus & $3.7 \pm 0.12$ & $1.9 \pm 0.1$ & $1 \pm 0.08$ \\
Bacillus subtilis & $2.9 \pm 0.11$ & $1.8 \pm 0.14$ & $1.4 \pm 0.15$ \\
Escherichia coli & $4 \pm 0.14$ & $1.7 \pm 0.11$ & $1.4 \pm 0.13$ \\
Pseudomonas aeruginosa & $3.7 \pm 0.14$ & $2.1 \pm 0.12$ & $1.2 \pm 0.13$ \\
& & $2.1 \pm 0.09$ & $1.4 \pm 0.14$ \\
& &
\end{tabular}

\pm Standard deviation

In this paper, TAE had shown better activity compared to DAE against gram positive bacteria (zone of inhibition in $\mathrm{cm}$, Table 4). The percentage of aldehyde group (70\%) seemed to have no effects on the antimicrobial properties of the extracts. The TAE extracts had more mannose than glucose. The DAE extracts had shown more or less equal activity against gram positive and gram negative bacteria. However, the DAE extracts had more glucose than mannose. In both of these extracts, it seemed galactose has no role. Glucose and mannose are epimeric. Ribose was present in the TAE extracts. However, no ribose was seen in the DAE extracts. In this study, we observe that extracts of TAE showed significant antimicrobial activity. The
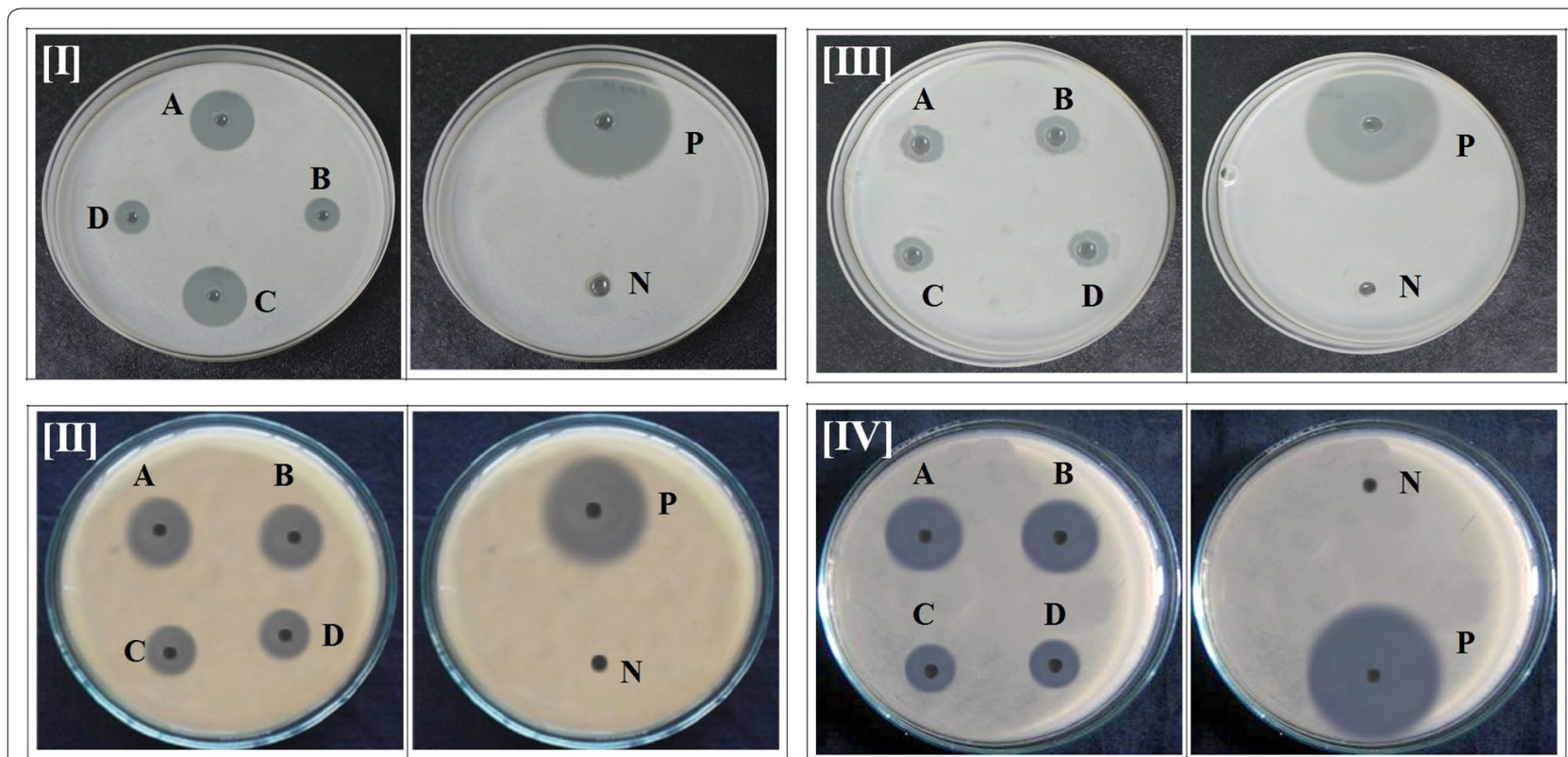

Fig. 12 Antimicrobial activity against [I] S. aureus of TAE (A,C) and DAE (B, D); [III] B.subtilis of TAE (A, C) and DAE (B, D); [III] E. coli of TAE (A, C) DAE (B, D); [IV] P. aureginosa of TAE (A, C) DAE (B, D) in comparison with (P) positive control as tetracycline (N) negative control as normal saline water 
results of antimicrobial activity of these polysaccharides as demonstrated herein are new and novel. The effects polysaccharides against human sugar levels are studied by some authors. But those polysaccharides (TAE and DAE) can also act as promising antimicrobial agents are not explored.

\section{Conclusions}

Compositions analysis for monosaccharide showed glucose, galactose and mannose residues are major compositions of the extract of TAE and a small amount of arabinose, ribose and xylose are also identified by GC-MS analysis. In DAE glucose and galactose are the main constituents. Also, the polysaccharides were morphologically nonporous as well as amorphous in nature. The antibacterial activities of TAE and DAE have a significant effect on human pathogenic bacteria. Among the both extracts, TAE showed better antimicrobial activity in comparison to the other extracts. So, extracts of TAE and DAE could be exploited for new potent antimicrobial agents.

\begin{abstract}
Abbreviations
GPC: gel permeation chromatoghrphy; SEM: scanning electron microscopy; XRD: X-ray diffractometer; EDX: energy-dispersive spectroscopy; FT-IR: the Fourier transform infrared spectra; TGA-DTA: thermo gravimetric and differential thermal analysis; GC-MS: gas chromatography mass spectrometry; TAE: T. ammi water extract; DAE: D. biflorus water extract; DCM: dichloromethane.
\end{abstract}

\section{Authors' contributions}

SB formulated the formulated the research idea and prepared the manuscript. MG contributed the TGA-DTA analysis and writing. RKB performed the GC-MS experiment. JG formulated, analyzed the research work. BKB reviewed and revised the manuscript for this journal. The manuscript has been approved by all authors and has never been published. All authors read and approved the final manuscript.

\section{Author details}

1 Department of Chemistry, Indian Institute of Engineering Science and Technology, Shibpur, Howrah, West Bengal, India. ${ }^{2}$ Department of Metallurgy and Materials Engineering, Indian Institute of Engineering Science and Technology, Shibpur, Howrah, West Bengal, India. ${ }^{3}$ Advanced Laboratory for Plant Genetic Engineering (ALPGE) and Advanced Technology Development Center (ATDC), Indian Institute of Technology (IIT), Kharagpur, Kharagpur, India. ${ }^{4}$ Research \& Education Development, Community Health Systems of South Texas, 3135 South Sugar Road, Edinburg, TX 78539, USA.

\section{Acknowledgements}

We acknowledge Prof. A. Mukherjee, Department of Botany, The University of Burdwan for the authentication of samples. We acknowledge Bankura Sammilani Medical College, Bankura, for providing access to their lab for testing antimicrobial activity. We acknowledge Centre for Research in Nanoscience and Nanotechnology, University of Calcutta, for lab support on SEM and EDX analysis.

\section{Competing interests}

The authors declare that they have no competing interests.

\section{Publisher's Note}

Springer Nature remains neutral with regard to jurisdictional claims in published maps and institutional affiliations.

Received: 9 August 2017 Accepted: 10 November 2017

Published online: 21 November 2017

\section{References}

1. Ekor M (2013) The growing use of herbal medicines: issues relating to adverse reactions and challenges in monitoring safety. Front Pharmacol 4:177. https://doi.org/10.3389/fphar.2013.00177

2. Sofowora A, Ogunbodede E, Onayade A (2013) The role and place of medicinal plants in the strategies for disease prevention. Afr J Tradit Complement Altern Med 10(5):210-229

3. Doering TL (2009) How sweet it is! cell wall biogenesis and polysaccharide capsule formation in Cryptococcus neoformans. Annu Rev Microbiol 63:223-247. https://doi.org/10.1146/annurev.micro.62.081307.162753

4. Bairwa R, Sodha RS, Rajawat BS (2012) Trachyspermum ammi. Pharmacogn Rev 6:56-60. https://doi.org/10.4103/0973-7847.95871

5. Basu S, Ganguly J (2016) A comparative bioefficacy of aqueous and methanolic extract of Trachyspermum ammi towards the antioxidant potentiality and electrochemical behaviour. AJOMC 1:17-21

6. Minija J, Thoppil JE (2002) Essential oil composition of Trachyspermum ammi (I.) sprague from South India. Indian J Pharm Sci 64:250-251

7. Ranjan B, Manmohan S, Singh SR, Singh RB (2012) Medicinal uses of Trachyspermum ammi: a review. Pharmacogn Rev 6:56-60

8. Qureshi AA, Kumar KE (2010) Phytochemical constituents and pharmacological activities of Trachyspermum ammi. Plant Arch 10:955-959

9. Srivastava M, Baby P, Saxena A (1999) GC-MS investigation and antimicrobial activity of the essential oil of Carum copticum Benth \& Hook. Acta Aliment 28:291-295

10. Garimella TS, Jolly Cl, Narayanan S (2001) In vitro studies on antilithiatic activity of seeds of Dolichos biflorus Linn. and rhizomes of Bergenia ligulata Wall. Phytother Res 15:351-355

11. Muthu AK, Sethupathy S, Manavalan R, Karar PK (2005) Hypolipidemic effect of methanolic extract of Dolichos biflorus Linn. in high fat diet fed rats. Indian J Exp Biol 43:522-525

12. Pant MC, Uddin I, Bhardwaj UR, Tewari RD (1968) Blood sugar and total cholesterol lowering effect of Glycine soja (Sieb and Zucc.), Mucuna pruriens (D.C.) and Dolichos biflorus (Linn.) seed diets in normal fasting albino rats. Indian J Med Res 56:1808-1812

13. Basu S, Maji P, Ganguly J (2015) Biosynthesis, characterisation and antimicrobial activity of silver and gold nanoparticles by Dolichos biflorus Linn seed extract. J Exp Nanosci 11:660-668. https://doi. org/10.1080/17458080

14. DuBois M, Gilles K, Hamilton J, Rebers P, Smith F (1956) Colorimetric method for determination of sugars and related substances. Anal Chem 28:350-356. https://doi.org/10.1021/ac60111a017

15. Swardeker JS, Sloneker JH, Jeans AR (1965) Quantitative determination of monosaccharides as their alditol acetates by gas-liquid chromatography. Anal Chem 37:1602-1604

16. Raveendran P, Fu J, Wallen SL (2006) A simple and "green" method for the synthesis of Au, Ag, and Au-Ag alloy nanoparticles. Green Chem 8:34-38

17. Tripathi A, Chandrasekaran N, Raichur AM, Mukherjee A (2009) Antibacterial application of silver nanoparticles synthesized by aqueous extract of Azadirachta indica (Neem) leaves. J Biomed Nanotechnol 5:93-98 